\title{
Imaging the stochastic microstructure and dynamic development of correlations in perpendicular artificial spin ice
}

\author{
Susan Kempinger, ${ }^{1, *}$ Robert D. Fraleigh, ${ }^{2}$ Paul E. Lammert, ${ }^{1}$ Sheng Zhang, ${ }^{3,4}$ Vincent H. Crespi, ${ }^{1}$ \\ Peter Schiffer, ${ }^{5}$ and Nitin Samarth $\oplus^{1, \dagger}$ \\ ${ }^{1}$ Department of Physics, The Pennsylvania State University, University Park, Pennsylvania 16802-6300, USA \\ ${ }^{2}$ Communications, Information and Navigation Office, Applied Research Laboratory, The Pennsylvania State University, \\ University Park, Pennsylvania 16802, USA \\ ${ }^{3}$ Materials Science Division, Argonne National Laboratory, 9700 South Cass Avenue, Argonne, Illinois 60439, USA \\ ${ }^{4}$ Advanced Science Research Center, The Graduate Center of the City University of New York, 85 Saint Nicholas Terrace, \\ New York, New York 10031, USA \\ ${ }^{5}$ Department of Applied Physics and Department of Physics, Yale University, New Haven, Connecticut 06520, USA
}

(Received 16 April 2019; revised manuscript received 12 September 2019; published 2 January 2020)

\begin{abstract}
We use spatially resolved magneto-optical Kerr microscopy to track the complete microstates of arrays of perpendicular anisotropy nanomagnets during magnetization hysteresis cycles. These measurements, along with our statistical methodology, allow us to disentangle the intertwined effects of nearest-neighbor interactions, disorder, and stochasticity on magnetization switching. We find that the nearest-neighbor correlations depend on both interaction strength and disorder. We also find that although the global characteristics of the hysteretic switching are repeatable, the exact microstate sampled is stochastic with the behavior of individual islands varying between nominally identical runs. These protocols provide another way of understanding the relationship between macrohistory and microhistory in artificial magnetic arrays.
\end{abstract}

DOI: 10.1103/PhysRevResearch.2.012001

Artificially structured lattices-artificial graphene [1], artificial skyrmion lattices [2], artificial spin ices [3-10], etc.have become popular platforms for studying complex collective phenomena in condensed matter because they allow systematic engineering and tuning of properties such as interaction strengths and defects to a degree far exceeding what is possible with naturally occurring lattices. Artificial spin ice (ASI) has allowed observation of magnetic monopoles and Dirac strings in well-controlled frustrated geometries [11-15], as well as access to the effects of thermal fluctuations $[16,17]$ and disorder $[18,19]$. Such systems are also interesting candidates for applications in neuromorphic computing and reconfigurable magnonics, which require an understanding of stochasticity and noise. Perpendicular ASI systems $[4,20]$ are particularly propitious in this context because polar magnetooptical Kerr effect (MOKE) microscopy allows complete in situ imaging of microstates and their evolution as an applied field is varied [21]. In this Rapid Communication, we combine MOKE microscopy with a statistical methodology to map out with microscopic detail the development of correlations in

\footnotetext{
*Present address: Department of Physics, North Central College, Naperville IL, USA

†nsamarth@psu.edu

Published by the American Physical Society under the terms of the Creative Commons Attribution 4.0 International license. Further distribution of this work must maintain attribution to the author(s) and the published article's title, journal citation, and DOI.
}

frustrated and unfrustrated magnetic arrays during the hysteretic magnetization reversal process, particularly around the coercive field. This approach contrasts with previous studies of the hysteresis loops of artificial spin ice systems which focus on the macrohistory of an array. In these prior studies, the development of the macrostate, characterized by aggregate quantities, is reproducible from one field cycle to another. Our focus on the microhistory of an array reveals that the evolution of its microstate during a field sweep is not reproducible from sweep to sweep. Although ambient temperature is very small compared to the naive magnetic energy scales, these systems are very sensitive to fluctuations of any sort when the external field is near the coercive field. Fluctuations can be amplified and locked in by the binary (Ising) character of island magnetization. The methodology developed here provides important insights into the magnetization reversal process and can be generalized to studies of other complex spin systems.

The samples studied in this Rapid Communication were patterned using electron beam lithography, with a standard liftoff of bilayer poly(methyl methacrylate)/ polymethylglutarimide (PMMA/PMGI) resist stack. All samples considered contain frustrated (kagome, triangular) and nonfrustrated (hexagonal, square) arrays. Lattice spacing ranges are $600-1000 \mathrm{~nm}$ for sample 1 , and $500-800 \mathrm{~nm}$ for samples 2 and 3. Scanning electron microscopy confirms island diameters of $400-450 \mathrm{~nm}$. Magnetic films of $\mathrm{Ti}(2 \mathrm{~nm}) / \operatorname{Pt}(10 \mathrm{~nm}) /[\mathrm{Co}(0.3 \mathrm{~nm}) / \operatorname{Pt}(1 \mathrm{~nm})]_{8}$ were deposited using dc sputtering at Argonne National Laboratory. We used superconducting quantum interference device (SQUID) magnetometry to confirm the strong perpendicular magnetic 
TABLE I. Physical, magnetic, and statistical properties of three different artificial spin ice samples.

\begin{tabular}{lcccccc}
\hline \hline & Diameter $(\mathrm{nm})$ & $M_{s}(\mathrm{~A} / \mathrm{m})$ & $B_{0}(500 \mathrm{~nm})(\mathrm{G})$ & $\sigma_{d}(\mathrm{G})$ & $\sigma_{h}(\mathrm{G})$ & Avg. overlap $(\%)$ \\
\hline Sample 1 & 400 & $3.46 \times 10^{5}$ & 3.61 & 15.70 & & \\
Sample 2 & 450 & $3.75 \times 10^{5}$ & 4.96 & 28.21 & $10.8 \pm 1.8$ & $87.7 \pm 1.1$ \\
Sample 3 & 425 & $3.46 \times 10^{5}$ & 4.09 & 17.28 & $9.8 \pm 0.9$ & $84.3 \pm 0.8$ \\
\hline
\end{tabular}

anisotropy of these films, as well as to measure the saturation magnetization for each film. Specific details on island size and magnetization properties for the samples considered are found in Table I.

Data are collected using an optimized polar MOKE imaging setup, described in detail elsewhere [21]. The complete microhistory of an array during a field sweep is obtained by in situ resolution of the microstate (magnetization state of every island) after each field step, as enabled by image processing and illustrated in Fig. 1. Since each island reverses magnetization only once during a field sweep, a microhistory $\alpha$ is encapsulated by the list of island switching fields: In sweep $\alpha$, island $i$ switches when $H_{\text {app }}=h_{i}^{\alpha}$. Although not distinguished notationally, up sweeps and down sweeps are treated separately, not combined in aggregate quantities or directly compared via correlation functions.

Reproducible aggregate aspects. We begin with the switching field distribution and the contribution of island interactions thereto. The total field experienced by an island comprises the applied field $H_{\text {app }}$ and a configuration-dependent contribution from other islands which broadens the distribution of observed (raw) switching fields. The semiempirical equation [21]

$$
\sigma=A K B_{0}(L)+\sigma_{d}
$$
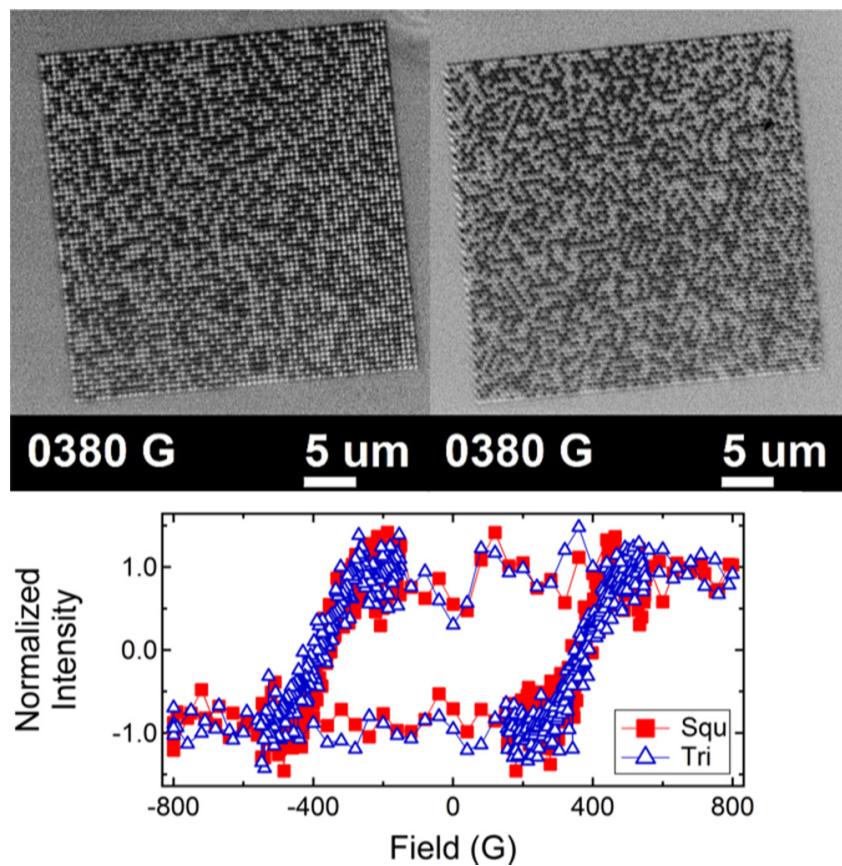

FIG. 1. Top: MOKE images recorded at $380 \mathrm{G}$ in an increasing the field sweep, near the coercive field, for 500-nm lattice spacing square (left) and triangular (right) arrays from sample 2. Bottom: Normalized hysteresis loops recorded using imaging MOKE for these arrays with intensity averaged over the entire array area. separates the observed width $\sigma$ of the switching field distribution into contributions of island interactions and static disorder, the latter presumably introduced by the lithography process. Here, $A$ is a constant, $K$ an effective coordination number, $B_{0}(L)$ the dipolar field of an island on its nearest neighbor at lattice spacing $L$, and $\sigma_{d}$ the static disorder. Additionally, from the microhistory we can directly calculate the $r$-neighborhood-corrected switching field $h_{i, r}=H_{\text {app }}+$ (field from up-to- $r$ th neighbors) in a point-dipole approximation, accounting for both the internal and external fields felt by an island when it switches. In this notation, $h_{i, 0}^{\alpha}$ is the raw switching field for sweep $\alpha$.

The top panel of Fig. 2 shows the distributions of the $r$-neighborhood-corrected switching fields $h_{*, r}^{\alpha}$ for a single sweep for a 500-nm square array from sample 2 for $0 \leqslant$ $r \leqslant 5$. (Similar results were obtained for sample 3.) These are the distributions of all aggregated islands, hence the " $*$ " subscript on $h$. The expected narrowing of the distribution as $r$
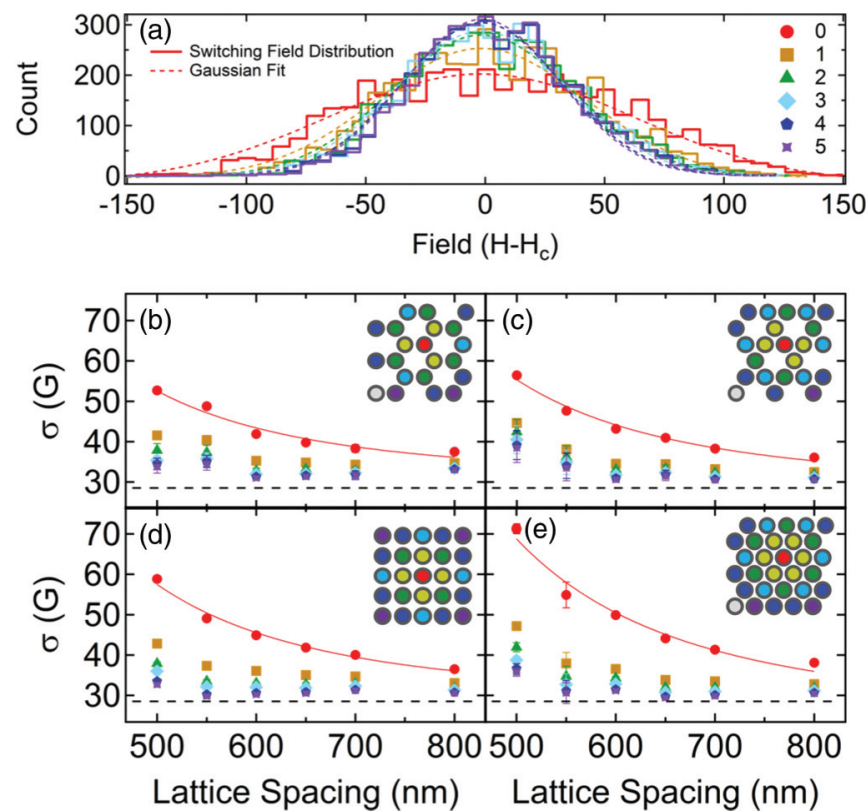

FIG. 2. (a) Switching field distribution and associated Gaussian fits, with switching fields calculated by removing dipolar effects from zero (as measured) to five nearest neighbors for a 500-nm square array (sample 2). Width of the Gaussian fits for (b) hexagonal, (c) kagome, (d) square, and (e) triangular as a function of lattice spacing taking into account increasing numbers of neighbors. Fits to Eq. (1) are shown as red lines, and disorder values from these fits are shown as black dashed lines. The inset images show a cartoon of the lattice geometry colored by target island (red) and neighbor number to match the colors on the graphs. A full set of neighbors is shown up to third nearest neighbor, along with a partial set of fourth and fifth nearest neighbors. 
increases (further-neighbor fields accounted for) is prominent. The lower panels of Fig. 2 show how the widths of the $h_{*, r}^{\alpha}$ distributions change with lattice spacing for different lattice types. The broadening in the raw $\left(h_{*, 0}^{\alpha}\right)$ distributions for different geometries is accounted for completely by the difference in effective coordination number. With increasing $r$, the width decreases, becoming independent of the lattice spacing and approaching the calculated value of disorder. The magnitude of the decrease is on the order of $A K B_{0}(L)$, with $K$ calculated considering $r$ neighbors, consistent with Eq. (1). This behavior agrees with previous studies pointing to the significance of long-range interactions to the behavior of artificial spin ice [22]. The analysis supports the treatment of islands as interacting point dipoles, wherein an island's neighbors influence its switching behavior by supplementing the external field with their net dipolar field strength.

While the $r$-neighborhood-corrected switching field distributions demonstrate an influence of islands on one another, they say nothing quantitative about correlations. We turn to these next. The average spin (magnetization) in an array during sweep $\alpha$ is $\left\langle S_{i}^{\alpha}\right\rangle_{i}$. Subscripts on averaging brackets indicate what is averaged over, and each spin takes a value +1 or $-1 .\langle S\rangle$ is a fairly reproducible function of external field (for the same sweep direction). To minimize finite-size effects in comparing different sweeps and different arrays, it is preferable to parametrize the macrohistory by magnetization $\langle S\rangle$ rather than the applied field. Thus, the nearest-neighbor spin correlation for sweep $\alpha$,

$$
\begin{aligned}
C_{S}^{\alpha}(\langle S\rangle) & =\left\langle S_{i}^{\alpha}\right\rangle_{i}\left\langle S_{j}^{\alpha}\right\rangle_{j}-\left\langle S_{i}^{\alpha} S_{j}^{\alpha}\right\rangle_{\alpha ; \mathrm{NN}} \\
& =\langle S\rangle^{2}-\left\langle S_{i}^{\alpha} S_{j}^{\alpha}\right\rangle_{\mathrm{NN}},
\end{aligned}
$$

is regarded as a function of $\langle S\rangle$. The sum is over all nearestneighbor pairs $(i, j)$ as indicated briefly by the $\mathrm{NN}$ subscript. $C_{S}$ is zero if spins are independently assigned values +1 or -1 with probabilities consistent with $\langle S\rangle$, and increases with the proportion of energetically preferred antiferromagnetic nearest-neighbor configurations with our chosen sign convention. Figures 3(a) and 3(b) show the evolution of $C_{S}^{\alpha}(\langle S\rangle)$ for up sweeps for the square and triangular arrays on sample 2 . The correlation increases and then decreases as the sample transitions from a saturated state, through zero magnetization to the oppositely saturated state. However, the correlation does not peak at zero magnetization. Rather, it increases and then peaks at some offset $\langle S\rangle$. This behavior indicates the importance of the quasidynamic switching path and the influence of island interactions on it. While the offsets are repeatable and observed in multiple samples, the data are too noisy to discern any clear trends in the values.

One anticipates that the maximum value of nearestneighbor antiferromagnetic correlation will increase with the strength of interactions, $B_{0}(L)$. Figure 3(c) shows that this expectation is borne out and that the dependence is roughly linear. Data for samples 1-3 are plotted in different colors. For each sample, the maximum value of $C_{S}(\langle S\rangle)$ is consistent among all geometries, indicating that the interactions are not sufficiently strong for the distinction between frustrated and unfrustrated geometry to manifest in the macrostate. However, there is a distinct variation in the correlations between samples, indicating that the interaction strength is an insuffi-
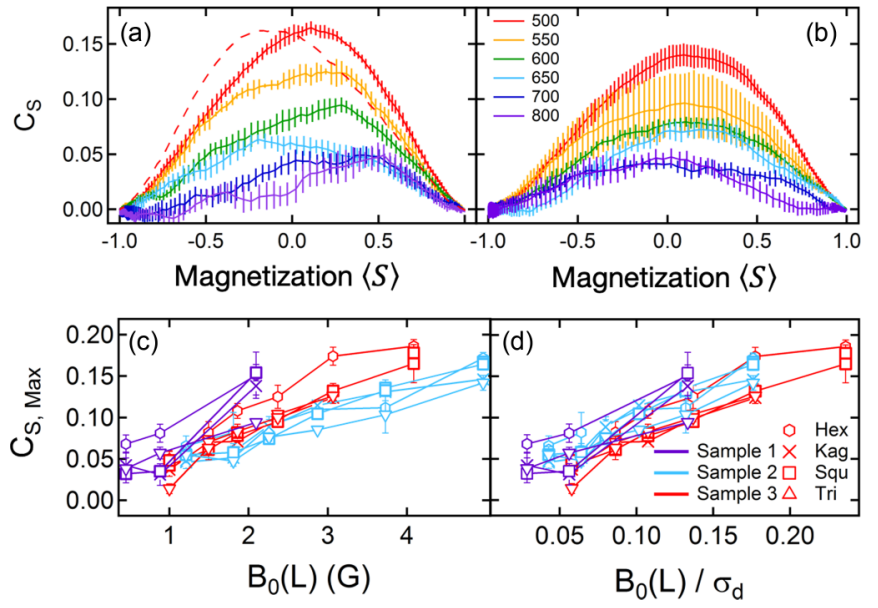

FIG. 3. Plots of $C_{S}(\langle S\rangle)$ for various lattice spacings of (a) square, and (b) triangular arrays from sample 2, for increasing values of applied field. The dashed line in (a) shows $C_{S}(\langle S\rangle)$ as the field is decreased to more clearly illustrate the asymmetry. (c) shows the maximum value of correlation as a function of the dipolar field of an island on its nearest neighbor (i.e., the interaction strength) for samples 1-3. (d) shows the same data as a function of the dipolar field scaled by the measured disorder in the system.

cient parameter to characterize these systems. Using instead the dimensionless ratio $B_{0}(L) / \sigma_{d}$ of interaction strength to static disorder as an independent variable, a significant, albeit partial, data collapse is obtained, as shown in Fig. 3(d). Quite reasonably, local ordering is enhanced by increasing interaction strength and hampered by increasing static disorder.

Microhistory stochasticity. The preceding discussion indicates that histories of the global quantities $\langle S\rangle$ and $C_{S}$, as well as distributions of switching fields $h_{i, 0}$ and $h_{i, r}$, are very similar run to run. A perfectly deterministic system, though, would have a reproducible microhistory, following exactly the same sequence of island switchings whenever subjected to the same external field sweep. A simple measure of nonreproducibility is the run-to-run switching field variance

$$
\sigma_{h}^{2}=\left\langle\left(h_{i}^{\alpha}-\overline{h_{i}}\right)^{2}\right\rangle_{i, \alpha},
$$

where $\overline{h_{i}}=\left\langle h_{i}^{\beta}\right\rangle_{\beta}$ is the run-averaged switching field of island $i$. The average in Eq. (3) is over islands in the array and seven to ten macroscopically identical hysteresis loops. In contrast to the aggregate switching field distributions displayed in Fig. 2, the run-to-run variance inherently involves an average over runs and involves subtraction of an island-dependent mean. Table I reports average values across all geometries of the run-to-run switching field standard deviation (the square root of the variance) $\sigma_{h}$ for samples 2 and 3 at lattice spacings above $650 \mathrm{~nm}$, of around $10 \mathrm{G} . \sigma_{h}$ increases with increasing interaction strength, maximizing at around $20 \mathrm{G}$ for the most strongly interacting arrays. These values are much less than the width of the aggregate switching field distribution because they measure different quantities. The aggregate switching field distribution measures the variation of $h_{i}$ throughout a lattice, while $\sigma_{h}$ measures the variation of the individual island's switching field around its mean value over a series of distinct runs. 

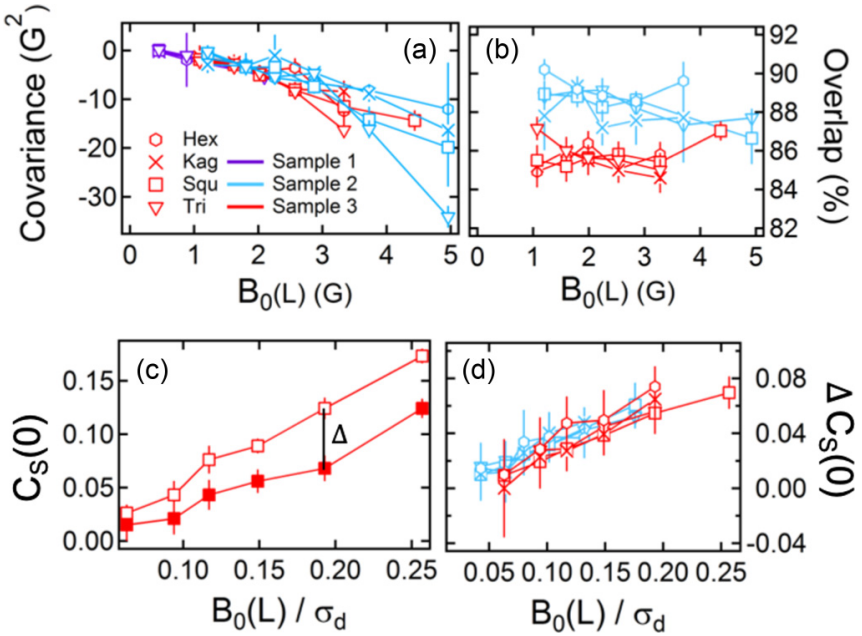

FIG. 4. (a) The covariance of switching fields between multiple runs for all three samples. (b) The average overlap at the coercive field between pairs of runs for samples 2 and 3. Overlap is defined as the percent of islands which are in the same state in both states considered. (c) The experimentally measured value of $C_{S}(0)$ (open squares) and the average value of $C_{S}(0)$ for randomly generated states with the experimentally measured overlap with the experimental state (solid squares), for a 500-nm square array from sample 3. The difference between these two curves is defined to be $\Delta C_{S}(0)$. (d) The average difference in correlation between the experimentally measured state and a state with the experimentally measured average overlap.

Island switching is significantly influenced by local environment; this is already clear from the switching field distributions in Fig. 2. An indication of how this influence contributes to microhistory variation is provided by the switching field covariance

$$
C_{h}=\left\langle\left(h_{i}^{\alpha}-\overline{h_{i}}\right)\left(h_{j}^{\alpha}-\overline{h_{j}}\right)\right\rangle_{\alpha ; \mathrm{NN}} .
$$

This quantity is plotted for all arrays in Fig. 4(a) as a function of interaction strength $B_{0}(L)$. That $C_{h}$ is negative conforms to expectations since if one island switches "early," it will increase the energy barrier for a neighbor to switch, due to the antiferromagnetic interactions. The arrays with the weakest interaction, although they show significant $\sigma_{h}$ (Table I), show no significant covariance. As the interactions are increased, the covariance between neighboring island's switching fields increases in magnitude. The increase in covariance also increases as a function of effective coordination number, similar to the switching field distribution broadening with effective coordination number. In fact, at these interaction strengths, the impact of array geometry can be described completely by the coordination of the array, rather than the presence or absence of frustration. The behavior at low interaction strength gives an indication of the intrinsic behavior of the islands, and the change with increasing interaction strength allows us to judge the impact of interactions. Because dynamics play a large role in the correlations of these systems, and there is some level of random variation that propagates through the lattice by neighbor interactions, it is likely that we will observe significant differences in the microstates.
To further characterize the (non)reproducibility of the microhistory, we examine the average overlap

$$
\overline{f_{=}}=\frac{1}{2}\left[1+\left\langle S_{i}^{\alpha} S_{i}^{\beta}\right\rangle_{i ; \alpha \neq \beta}\right]
$$

at zero magnetization, $\langle S\rangle=0$. The average overlap is the fraction of islands which are in the same state in a randomly chosen pair of distinct runs. Calculated values for samples 2 and 3 are plotted in Fig. 4(b) and range from $84 \%$ to $90 \%$. Sample 2 has a consistently larger overlap than sample 3, which is reasonable since $\sigma_{h}$ is similar for the two samples while $\sigma_{d}$ is larger for sample 2. A larger ratio of $\sigma_{d} / \sigma_{h}$ implies that each island has access to a smaller subset of the switching region, increasing the number of islands in the same state at any given point in the switching process.

One may wonder whether an average overlap approaching $90 \%$ is enough by itself to explain the observed macrohistory repeatability. A simple numerical experiment shows this is not the case. Starting from one specific $\langle S\rangle=0$ microstate, we randomly select a fraction $1-\overline{f_{=}}$of islands, flip them, and calculate the change $\Delta C_{S}(0)$ of the nearest-neighbor correlation [see Fig. 4(c)]. Average values of $\Delta C_{S}(0)$ for 1000 repetitions of this experiment are plotted in Fig. 4(d). The drop in $C_{S}$ is significantly greater than the standard deviation of the distribution over runs, hence one concludes that there is more to the correlations than simply the overlap. Indeed, one may calculate that if microstate $S_{i}^{\prime}$ is obtained from $S_{i}$ by independently flipping spins with probability $1-\overline{f_{=}}$, that the nearest-neighbor correlation of the new microstate has an expectation value

$$
\left\langle S_{i}^{\prime} S_{j}^{\prime}\right\rangle_{\mathrm{NN}}=\left(1-2 \overline{f_{=}}\right)^{2}\left\langle S_{i} S_{j}\right\rangle_{\mathrm{NN}} .
$$

The origin(s) of microhistory stochasticity are not clear. Noise arising from the experimental setup, for instance, in the power supply or magnet, seem unlikely to be responsible since such influences would be uniform across the sample; the magnetic field is quite homogeneous over our small field of view. However, the significant run-to-run switching field covariance shows that the stochasticity is at least strongly affected by local conditions. Prima facie, one expects thermal fluctuations to be completely negligible; the energy scale of room-temperature $k_{B} T$ equals the magnetic energy of an island in a field of order $10^{-1} \mathrm{G}$, about $5 \%$ the field step size, which should lead to a high thermal stability at room temperature. However, near the coercive field, thermal fluctuations can be surprisingly significant in understanding the behavior of nanomagnetic systems [23,24]. A non-negligible fraction of islands might be caused to switch in a slightly different field by a thermal fluctuation in a given run, and the "misstep" would then be amplified and propagated by island interactions. One might expect these propagated missteps to lead to a decrease in the zero magnetization overlap as the interactions are increased. However, we observe that the overlap is insensitive to interactions, possibly because only a subset of islands is susceptible to thermal fluctuations at any given field step. Any island with a coercivity that is not sufficiently close to a given field is constrained to remain stable in its moment orientation at that field in all runs. 
In conclusion, MOKE microscopy allows us to measure complete microhistories of perpendicular artificial spin ice, providing a direct and precise quantification of the role of island interactions and stochasticity on the macrohistory during magnetization reversal. These insights suggest other seemingly stable systems of interest for applications may show room-temperature microstate stochasticity. Our results provide another protocol for future studies of the complex relationship between the microhistory and macrohistory of artificial spin ices and other artificially patterned nanomagnetic arrays.

S.K., P.S., and N.S. acknowledge support from US Department of Energy, Office of Basic Energy Sciences, Materials Sciences and Engineering Division under Grant No. DESC0010778. P.E.L. and V.H.C. acknowledge support from the National Science Foundation MRSEC program under Grant No. DMR-1420620.
[1] L. Ládvorník, M. Orlita, N. A. Goncharuk, L. Smrčka, V. Novák, V. Jurka, K. Hruška, Z. Výborný, Z. R. Wasilewski, M. Potemski, and K. Výborný, New J. Phys. 14, 053002 (2012).

[2] D. A. Gilbert, B. B. Maranville, A. L. Balk, B. J. Kirby, P. Fischer, D. T. Pierce, J. Unguris, J. A. Borchers, and K. Liu, Nat. Commun. 6, 8462 (2015).

[3] R. F. Wang, C. Nisoli, R. S. Freitas, J. Li, W. McConville, B. J. Cooley, M. S. Lund, N. Samarth, C. Leighton, V. H. Crespi, and P. Schiffer, Nature (London) 439, 303 (2006).

[4] S. Zhang, J. Li, I. Gilbert, J. Bartell, M. J. Erickson, Y. Pan, P. E. Lammert, C. Nisoli, K. K. Kohli, R. Misra, V. H. Crespi, N. Samarth, C. Leighton, and P. Schiffer, Phys. Rev. Lett. 109, 087201 (2012).

[5] L. J. Heyderman and R. L. Stamps, J. Phys.: Condens. Matter 25, 363201 (2013).

[6] I. Gilbert, C. Nisoli, and P. Schiffer, Phys. Today 69(7), 54 (2016).

[7] J. P. Morgan, C. J. Kinane, T. R. Charlton, A. Stein, C. Sánchez-Hanke, D. A. Arena, S. Langridge, and C. H. Marrows, AIP Adv. 2, 022163 (2012).

[8] G. M. Wysin, W. A. Moura-Melo, L. A. S. Mól, and A. R. Pereira, New J. Phys. 15, 045029 (2013).

[9] I. Gilbert, G. W. Chern, B. Fore, Y. Lao, S. Zhang, C. Nisoli, and P. Schiffer, Phys. Rev. B 92104417 (2015).

[10] Y. Li, G. Paterson, G. M. Macauley, F. S. Nascimento, C. Ferguson, S. A. Morley, M. C. Rosamond, E. H. Linfield, D. A. MacLaren, R. Macedo, C. H. Marrows, S. McVitie, and R. L. Stamps, ACS Nano 92, 104417 (2018).

[11] S. Ladak, D. E. Read, W. R. Branford, and L. F. Cohen, New J. Phys. 13, 063032 (2011).
[12] E. Mengotti, L. J. Heyderman, A. F. Rodríguez, F. Nolting, R. V. Hügli, and H.-B. Braun, Nat. Phys. 7, 68 (2011).

[13] C. Phatak, A. K. Petford-Long, O. Heinonen, M. Tanase, and M. De Graef, Phys. Rev. B 83, 174431 (2011).

[14] L. A. Mól, R. L. Silva, R. C. Silva, A. R. Pereira, W. A. MouraMelo, and B. V. Costa, J. Appl. Phys. 106, 063913 (2009).

[15] Y. Perrin, B. Canals, and N. Rougemaille, Nature (London) 540, 410 (2016).

[16] V. Kapaklis, U. B. Arnalds, A. Farhan, R. V. Chopdekar, A. Balan, A. Scholl, L. J. Heyderman, and B. Hjörvarsson, Nat. Nanotechnol. 9, 514 (2014).

[17] A. Farhan, P. M. Derlet, A. Kleibert, A. Balan, R. V. Chopdekar, M. Wyss, L. Anghinolfi, F. Nolting, and L. J. Heyderman, Nat. Phys. 9, 375 (2013).

[18] Z. Budrikis, in Solid State Physics, edited by R. E. Camley, and R. L. Stamps (Elsevier, Amsterdam, 2014), Vol. 65, pp. 109-236.

[19] K. K. Kohli, A. L. Balk, J. Li, S. Zhang, I. Gilbert, P. E. Lammert, V. H. Crespi, P. Schiffer, and N. Samarth, Phys. Rev. B 84, 180412(R) (2011).

[20] E. Mengotti, L. J. Heyderman, A. Bisig, A. Fraile Rodríguez, L. Le Guyader, F. Nolting, and H. B. Braun, J. Appl. Phys. 105, 113113 (2009).

[21] R. D. Fraleigh, S. Kempinger, P. E. Lammert, S. Zhang, V. H. Crespi, P. Schiffer, and N. Samarth, Phys. Rev. B 95, 144416 (2017).

[22] I. A. Chioar, N. Rougemaille, A. Grimm, O. Fruchart, E. Wagner, M. Hehn, D. Lacour, F. Montaigne, and B. Canals, Phys. Rev. B 90, 064411 (2014).

[23] H. B. Braun, Phys. Rev. Lett. 71, 3557 (1993).

[24] Y. Telepinsky, O. Sinwani, V. Mor, M. Schultz, and L. Klein, J. Appl. Phys. 119, 083902 (2016). 